\title{
Severe periconceptional life events and the sex ratio in offspring: follow up study based on five national registers
}

\author{
Dorthe Hansen, Henrik Møller, Jørn Olsen
}

John F Kennedy Institute, $\mathrm{Gl}$ Landevej 7, 2600 Glostrup, Denmark Dorthe Hansen paediatrician

Centre for Research in Health and Social Statistics, Danish National Research

Foundation,

Sejrøgade 11, 2100

Copenhagen $\varnothing$,

Denmark

Henrik Møller

head

Danish

Epidemiology

Science Centre,

Vennelyst

Boulevard 6, 8000

Aarhus C, Denmark Jørn Olsen

professor

Correspondence to: Dr Hansen d209325@inet. uni2.dk

BMJ 1999;319:548-9
Exposure to smog, floods, ${ }^{1}$ or earthquakes ${ }^{2}$ has been associated with a lower sex ratio (proportion of males) in offspring conceived at the time of the exposure episode. We hypothesised that exposure to severe life events before and during the periconceptional period might be associated with a decline in the sex ratio.

\section{Methods and results}

All Danish women who gave birth from 1 January 1980 to 31 December 1992 were identified by the population based medical birth registry. By linking personal identification numbers to the Danish statistical office's fertility database, we identified all the women's partners and older children. A partner was defined as the father of the child or the partner with whom the woman was cohabiting on 1 January in the year of birth. Severe life events were defined as death or admission to hospital for cancer (codes 140 to 209 (excluding 208 polycythemia vera) of the international classification of diseases, eighth revision) or acute myocardial infarction (code 410) in the partner or older children. By linking the personal identification numbers to the mortality registry and to the national registry of patients, we identified all deaths and all admissions to hospital of partners and children between 1978 and 1992.

We identified all women exposed to severe life events in the year of birth and the previous year but included only the women exposed before the second trimester. Women with diabetic partners were excluded, as it has been reported that the sex ratio in their offspring is higher. The time of exposure was recorded as the date of death or first admission to hospital. Information on the possible confounding variables was obtained from the registries.

The exposed cohort consisted of 3072 singleton pregnancies exposed to severe life events and a control cohort of 20337 singleton pregnancies randomly selected among all pregnancies without such exposure in the observation period. Of all the offspring, 945 had congenital malformations, chromosomal abnormalities, or hereditary diseases.

Using logistic regression analysis, we found that the proportion of boys was $49.0 \%$ in the exposed group and $51.2 \%$ the control group, giving a crude overall lower ratio between the two sex odds (sex odds ratio) of 0.92 and an adjusted ratio of 0.91 (95\% confidence interval 0.84 to 0.99 ) (table). Exclusion of offspring whose mothers were exposed during the first trimester and all offspring with congenital malformations gave similar results (data not shown).

The nearer the exposure was to conception, the lower the overall sex odds ratio (test for trend $\mathrm{P}=0.013)$ and the lower the sex ratio for exposure by an older sibling only $(\mathrm{P}=0.035)$ and for exposure by death of a partner only $(\mathrm{P}=0.016)$.

\section{Comment}

The results show that severe life events may reduce the sex ratio, especially for exposures around the time of conception, because of differential conception or

Adjusted sex odds ratios and percentage of boys as function of severe life events before and in periconceptional period and stratified for timing of exposure

\begin{tabular}{|c|c|c|c|}
\hline Type of cohort & Numbers & Boys $(\%)$ & Sex odds ratio ( $95 \%$ confidence interval)* \\
\hline Controls & 20337 & 51.2 & 1.00 \\
\hline Exposed women: & 3072 & 49.0 & $0.91(0.84$ to 0.99$)$ \\
\hline Exposed 13-16 months before† & 71 & 52.1 & $1.04(0.65$ to 1.69$)$ \\
\hline Exposed 7-12 months before $†$ & 789 & 49.6 & $0.94(0.81$ to 1.08$)$ \\
\hline Exposed 0-6 months before† & 1922 & 48.9 & $0.91(0.83$ to 1.01$)$ \\
\hline Exposed first trimester & 290 & 46.0 & 0.81 (0.64 to 1.02$)$ \\
\hline \multicolumn{4}{|l|}{ Source of exposure: } \\
\hline Older sibling: & 2661 & 49.4 & $0.93(0.85$ to 1.01$)$ \\
\hline Exposed 13-16 months before $\dagger$ & 56 & 55.4 & $1.18(0.70$ to 2.00$)$ \\
\hline Exposed 7-12 months before $†$ & 693 & 50.8 & 0.98 (0.84 to 1.15$)$ \\
\hline Exposed 0-6 months before $\dagger$ & 1771 & 49.1 & $0.92(0.83$ to 1.02$)$ \\
\hline Exposed first trimester & 141 & 43.6 & $0.74(0.53$ to 1.03$)$ \\
\hline Partners & 411 & 46.5 & $0.83(0.68$ to 1.01$)$ \\
\hline \multicolumn{4}{|l|}{ Type of life event: } \\
\hline Death & 2575 & 49.0 & $0.92(0.84$ to 1.00$)$ \\
\hline Exposed 13-16 months before & 44 & 54.7 & 1.15 (0.63 to 2.08$)$ \\
\hline Exposed $7-12$ months before $\dagger$ & 650 & 50.5 & 0.97 (0.83 to 1.14$)$ \\
\hline Exposed 0-6 months before $\dagger$ & 1718 & 49.2 & 0.92 (0.83 to 1.02$)$ \\
\hline Exposed first trimester & 163 & 40.6 & 0.65 (0.47 to 0.89$)$ \\
\hline Cancer & 426 & 48.1 & 0.88 (0.73 to 1.07$)$ \\
\hline Acute myocardial infarction & 71 & 49.6 & 0.94 (0.59 to 1.59$)$ \\
\hline
\end{tabular}

*Sex ratio of exposed/sex ratio of non-exposed; and adjusted for maternal and partners' ages as well as for school education, maternal parity (nulliparous $v$ multiparous), previous spontaneous abortions (yes/no), residence (capital, major city, or rural), and congenital malformation in offspring (yes/no). †Before first day of last menstruation. 
differential abortion of male embryos. The study cohort was large, and the registration of death, cancer, and the offsprings' sex is known to be accurate and complete in the registers used. The results are consistent with results for natural disasters. ${ }^{12}$

The study has some limitations. The exact dates of the actual stress exposure may in most cases have started long before our date of exposure. For this reason, we also included women exposed during the first trimester. More factors may have contributed to a dilution of the effect-for example, other stressors may have affected both the women in the exposed group and those in the control group, and the partners may not have been the father of the offspring in all cases.

Psychological stress related to severe life events may alter the sex ratio through changes in sexual activity, changes in hormones around the time of conception, ${ }^{3}$ reduced semen quality, ${ }^{2}{ }^{4}$ or an increased rate of early male abortion. ${ }^{5}$

DH and JO also work, as paediatrician and professor respectively, at the Danish Epidemiology Science Centre, Statens Serum Institut, in Copenhagen.
Contributors: DH and JO contributed to the idea, design, analysis, interpretation of data, and writing of the paper. HM contributed to the interpretation of the data and critically reviewed the draft paper. All authors will act as guarantors for the paper.

Funding: Danish Medical Research Council, Health Insurance Foundation, Dagmar Marshall Foundation, and Danish Medical Association Research Foundation. The activities of the Danish Epidemiology Science Centre are financed by a grant from the Danish National Research Foundation.

Competing interests: None declared.

1 Lyster WR. Altered sex ratio after London smog of 1952 and the Brisbane flood of 1965.J Obstet Gynecol Br Commonwealth 1974;81:626-31.

2 Fukuda M, Fukuda K, Shimizu T, Møller H. Decline in sex ratio at birth after Kobe earthquake. Human Reprod 1998;13:2321-2.

3 James WH. Evidence that mammalian sex ratios at birth are partially controlled by parental hormones levels at the time of conception. $J$ Theor Biol 1996;180:271-86.

4 Fenster L, Katz DF, Wyrobek AJ, Pieper C, Rempel DM, Oman D, et al. Effects of psychological stress on human semen quality. $J$ Androl 1997; 18:194-202.

5 Pratt NC, Lisk RD. Effects of social stress during early pregnancy on litter size and sex ratio in the golden hamster (Mesocricetus auratus).J Reprod Fert 1989;87:736-69.

(Accepted 31 March 1999)

\section{Fatal erythroderma associated with pentostatin}

H S Ghura, A J Carmichael, D Bairstow, R Finney, Departments of Dermatology and Haematology, North Tees General Hospital, Stockton on Tees TS19 8PE

A 59 year old woman presented with weight loss and hepatosplenomegaly. Her medical history was unremarkable with no history of skin disease. She was not taking drugs systemically and gave no history of allergy. Full blood count showed a haemoglobin concentration of $142 \mathrm{~g} / \mathrm{l}$, a white cell count of $164 \times 10^{9} / \mathrm{l}$, and a platelet count of $105 \times 10^{9} /$. The results of cell marker and morphological studies confirmed the diagnosis to be $\mathrm{T}$ cell chronic prolymphocytic leukaemia. Her renal function was normal.

She was treated with chlorambucil $10 \mathrm{mg} /$ day However, despite treatment for 12 weeks, her white cell count rose to $324 \times 10^{9} / 1$ and her haemoglobin concentration fell to $60 \mathrm{~g} / 1$ so that she had to have a transfusion. In view of her failure to respond, chlorambucil was discontinued and she was given a single intravenous dose of pentostatin $4 \mathrm{mg} / \mathrm{m}^{2}$. Five days later she was admitted with a fever and diffuse erythema on her legs and back which blanched only partially on diascopy (firm pressure applied with a glass slide). The rash initially extended on to her abdomen, reaching its maximum extent nine days after the pentostatin injection. The rash then exfoliated and resolved over the next week. She was treated empirically at admission with intravenous flucloxacillin and ciprofloxacin. Blood cultures were subsequently found to be negative.

After an initial fall in white cell count to $128 \times 10^{9} / 1$, her count began to rise again to $244 \times 10^{9} / 1$ within two weeks of the pentostatin injection. Treatment with a further intravenous injection of pentostatin $4 \mathrm{mg} / \mathrm{m}^{2}$ was started after discussion with colleagues and in the absence of an alternative treatment or previous reports of pentostatin causing severe dermatological adverse reactions. The rash recurred within two days but was more extensive, such that she had erythroderma, had reactive oedema, and was clinically and biochemically dehydrated. Despite intravenous rehydration, her condition deteriorated rapidly and she died of presumed septicaemia five days after readmission.

Pentostatin is a product of Streptomyces antibioticus that inhibits adenosine deaminase. It is highly effective in hairy cell leukaemia as well as other low grade lymphoid tumours, including chronic lymphatic leukaemia, prolymphocytic leukaemia, low grade non-Hodgkin's lymphoma, and cutaneous $\mathrm{T}$ cell lymphoma. ${ }^{1}$

A range of rashes has been described with pentostatin, including a photosensitive, erythematous, papular, and vesiculobullous eruption. ${ }^{2}$ We are unaware of previous reports of erythroderma with pentostatin. Conditions associated with altered immune function, such as AIDS, predispose to adverse drug reactions in the skin. ${ }^{3}$ Pentostatin suppresses CD4 and CD8 counts, interleukin 2 secretion, and T cell mitogen response, ${ }^{1}$ perhaps accounting for the particularly high incidence of dermatological adverse reactions with the use of pentostatin, which was as high as $43 \%$ in one series. ${ }^{2}$

Given the potentially life threatening nature of erythroderma, ${ }^{4}$ particularly in an immunocompromised patient, we think that pentostatin should be added to the list of drugs capable of triggering this severe adverse reaction in the skin. ${ }^{5}$ In addition, we would strongly discourage others from rechallenging patients who have previously had an adverse skin reaction with pentostatin.

We thank Dr E Matutes, consultant haematologist, Royal Marsden Hospital, for her helpful advice in this case.

1 Saven A, Piro L. Newer purine analogues for the treatment of hairy-cell leukemia. N Engl J Med 1994;330:691-7.

2 McEvoy GK. The American hospital formulary service. Bethesda, MA: American Society of Health System Pharmacists, 1997.

3 Coopman SA, Stern RS. Cutaneous drug reactions in human immunodeficiency virus infection. Arch Dermatol 1991;127:714-7.

4 King LE. Erythroderma. Arch Dermatol 1994;130:1545-7.

5 Breathnach SM, Hintner M. Adverse drug reactions in the skin. Oxford: Blackwell Scientific Publications, 1992. 